CERN-OPEN-2008-010

DESY-08-040

FERMILAB-PUB-08-077-BSS

SLAC-PUB-13199

April 7, 2008

\title{
Information Resources in High-Energy Physics Surveying the Present Landscape and Charting the Future Course
}

\author{
Anne Gentil-Beccot, Salvatore Mele \\ CERN, European Organization for Nuclear Research \\ CH1211, Genève 23, Switzerland \\ Annette Holtkamp \\ Deutsches Elektronen-Synchrotron DESY \\ Notkestraße 85, D-22607 Hamburg, Germany \\ Heath B. O'Connell \\ Fermilab Library MS $109^{1}$ \\ P.O. Box 500, Batavia, Illinois 60510 USA \\ Travis C. Brooks \\ SLAC, Stanford Linear Accelerator Center Library/SPIRES Databases ${ }^{2}$ \\ Stanford University, Stanford, CA 94309, USA
}

\begin{abstract}
Access to previous results is of paramount importance in the scientific process. Recent progress in information management focuses on building e-infrastructures for the optimization of the research workflow, through both policy-driven and user-pulled dynamics. For decades, High-Energy Physics (HEP) has pioneered innovative solutions in the field of information management and dissemination. In light of a transforming information environment, it is important to assess the current usage of information resources by researchers and HEP provides a unique test-bed for this assessment. A survey of about $10 \%$ of practitioners in the field reveals usage trends and information needs. Community-based services, such as the pioneering arXiv and SPIRES systems, largely answer the need of the scientists, with a limited but increasing fraction of younger users relying on Google. Commercial services offered by publishers or database vendors are essentially unused in the field. The survey offers an insight into the most important features that users require to optimize their research workflow. These results inform the future evolution of information management in HEP and, as these researchers are traditionally "early adopters" of innovation in scholarly communication, can inspire developments of disciplinary repositories serving other communities.
\end{abstract}

\footnotetext{
${ }^{1}$ Operated by Fermi Research Alliance, LLC under Contract No. DE-AC02-07CH11359 with the United States Department of Energy

${ }^{2}$ Work partly supported by Department of Energy contract DE-AC02-76SF00515
} 


\section{Introduction}

High-Energy Physics (HEP), also known as Particle Physics, has a long record of innovation in scholarly communication. Half a century ago, theoretical physicists and experimental collaborations mailed to their peers hundreds, even thousands, of copies of their manuscripts. This occurred at the time of submission to peer-reviewed journals, whose speed in disseminating scientific information was deemed to be insufficient for the speed at which the field was evolving [1]. This practice led to the creation of the first electronic catalog for gray literature, later evolving into a catalog of the entire subject literature: the SPIRES database [2].

In the last two decades, crucial innovation in scholarly communication emerged from the HEP community, ranging from the invention of the world-wide web at CERN [3] to the inception of arXiv, the first and archetypal repository [4]. The onset of the web gave SPIRES the honor to be the first web server in America and the first database on the web [5]. More recently the HEP community inspired the development of Invenio, one of the first open-source digital library software packages [6], currently used for repositories in many fields.

Thanks to this suite of user-driven innovations, HEP scholars have used a variety of dedicated, field-specific "information resources". For many decades these have been run by large research institutions as a natural evolution of more conventional library services. At their inception, these resources often provided unique services, or were tailored specifically to the needs of the HEP community. Many of these services still exist and still provide information that cannot be obtained in any other way.

For many years now almost all journal literature has been electronically available, the entire web is readily searchable, and commercial online databases provide metadata about all scientific literature. In addition, online services are changing more and more rapidly as new tools are developed and new ways of interacting with users evolve. In light of this fast-changing world, it is important to assess the usage by HEP researchers of HEP-specific information resources. Such a study serves two purposes: within the field, it informs on the need for such community-based resources and their real role in the present internet landscape, inspiring their future evolution; globally, it provides an in-depth case study of the impact of discipline-based information resources, as opposed to institution-based information resources or cross-cutting (commercial) information platforms. This information is particularly relevant in light of recent worldwide moves towards self-archiving of research results at the institutional or disciplinary level, and the need to effectively incorporate these resources in the research workflow.

A survey of HEP scholars was designed and deployed in order to provide a unique insight into their information needs and the way their research workflow includes information discovery and retrieval. Its results are presented in this Article. The Article first describes the current landscape of HEP information resources (Section 2), then presents the survey methodology and the demographics of the respondents (Section 3). Two sets of results are presented and discussed: the information resources preferred by HEP researchers (Section 4) and their appreciation of the relative importance of possible features of information resources (Section 5). The survey also provides additional information on user requirements for the future of information resources (Section 6). After the conclusions of the study (Section 7), an Appendix presents some of the most inspiring free-text answers charting the future of information provision in this field. 


\section{The Landscape of HEP Information Resources}

Several information resources serve the needs of HEP researchers, as summarized in the following.

- arXiv [7]. arXiv is the archetypal repository. It was conceived in 1991 by Paul Ginsparg, then at the Los Alamos National Laboratory in New Mexico, and is now hosted at Cornell University in New York. It evolved a four-decade old tradition of HEP preprint circulation into an electronic based system, offering all scholars a level playing-field from which to access and disseminate information. Today arXiv counts nearly 500,000 preprints and has grown outside the field of HEP, becoming the reference repository for many diverse disciplines beyond physics, from mathematics to some areas of biology. arXiv functions almost solely as a repository, with little emphasis on sophisticated searching or curation of bibliographic information.

- CDS [8]. The CERN Document Server (CDS) was conceived in the late 90s at CERN, the European Organization for Nuclear Research in Geneva, Switzerland for the management of scientific information at the laboratory [9]. It has a double role. On one side, it is CERN's institutional repository, with a mission to archive and disseminate CERN results in the fields of experimental and theoretical physics as well as accelerator and information technologies. On the other side, given the central role of CERN in research in the field, it expanded to also offer a gateway to HEP information at large, indexing the content of major journals and harvesting full-text from many preprint servers, with most of the content coming from arXiv. These efforts are more limited in scope and time than those at SPIRES, discussed below. CDS is based on the Invenio open-source digital library software [6]. CDS counts about 1 million records and 500,000 full text-documents, as well as a growing multimedia collection.

- SPIRES [10]. SPIRES has provided a metadata-only search engine for all literature in the field for over 30 years. It is hosted at SLAC, the Stanford Linear Accelerator Center in California, and jointly compiled together with DESY, the Deutsches Elektronen-Synchrotron in Hamburg, Germany, and Fermilab, the Fermi National Accelerator Laboratory in Illinois. SPIRES adds citation data, keywords, classifications and authors with their institutional affiliations to the basic data that is harvested from various sources of physics literature. Today SPIRES has grown to include 750,000 records. SPIRES functions primarily as a gateway to all information in the field, providing context and consolidation for other data sources. SPIRES also provides a corrections and additions capability so that authors and users can correct errors they might find. In addition, other information of interest to the HEP community is offered at the SPIRES site via databases of jobs, conferences, people and institutions [11].

A fourth, community-based, information resource also serves the HEP community, even though it was originally designed for the astronomy and astrophysics communities.

- ADS [12]. The Astrophysics Data System (ADS) is a digital library portal for researchers in astronomy and physics, operated by the Smithsonian Astrophysical Observatory at Harvard under a NASA grant. It serves as a portal to astronomy and 
astrophysics data as well as bibliographic records. It offers highly customizable query forms and gives access to full-text scans of much of the astronomical literature which can be browsed or searched via a full-text search interface.

The relations that exist between the different HEP information resources are relevant to understanding the degree of interoperability and complementarity between them. In addition they help shed light on both the workflow of HEP researchers and the findings of the survey. The main relations are as follows:

- ADS \& arXiv. ADS maintains a database of all arXiv content relevant to HEP, offering additional services such as highly customizable e-mail alerts or RSS feeds.

- CDS \& arXiv. CDS carries all arXiv content relevant to HEP, with targeted curation effort devoted to matching preprints with information on publication reference, conference contributions, experimental collaborations, and the use of local authority files for author disambiguation.

- SPIRES \& arXiv. Because of their similar histories and mostly non-overlapping functions, SPIRES and arXiv could be considered as a single system. arXiv functions as the back-end data storage, as well as managing all of the complexities of submission. SPIRES provides a front-end interface, as well as giving further context to the arXiv submissions by matching them with published literature and adding citation, keywords and other data ${ }^{3}$. Examples of their symbiosis include the fact that all of the arXiv content of HEP relevance is indexed in SPIRES and arXiv relies on SPIRES for tasks like citation analysis.

Like virtually everyone else with internet access, HEP scholars also use Google [13] and Google Scholar [14] as information resources. One of the targets of this study is indeed to assess the penetration of these resources in the HEP scholarly-communication landscape. It is important to remark that arXiv and SPIRES have let their content be harvested by Google and then partly organized in Google Scholar.

\section{Survey Methodology and Demographics}

The data discussed in this Article were obtained by an anonymous on-line survey widely distributed in the HEP community. The survey ran for 6 weeks, from April 30, 2007 to June 11, 2007, and collected 2,115 responses. The number of respondents can be compared with the number of HEP physicists active in 2006, which is about 20,000 [15], or the number of authors who have published an article listed in SPIRES in the last decade, which is between 30,000 and 40,000, depending on how one handles similar names. It can be safely concluded that between $5 \%$ and $10 \%$ of the HEP community participated in the survey. This incredible rate of participation was further enhanced by the fact that $90 \%$ of the respondents wrote some optional free-text comments in addition to the required "radio-button" selections, and $73 \%$ responded to optional lengthier questions. The engagement of the community is further

\footnotetext{
${ }^{3}$ This is an oversimplified view, as arXiv.org does provide some searching capabilities and, since not all literature is submitted to arXiv nor is all arXiv content HEP related, the data sets in the two services are not identical.
} 
signified by the fact that about half of the respondents asked to be informed via e-mail of the results of the poll.

This survey was promoted within the HEP community by e-mailing members of major experimental collaborations, users of major laboratories and authors of a major journal of the field: the Journal of High Energy Physics. A link to the survey was also distributed for a week as a heading in the popular daily e-mail alerts of arXiv. Prominent notices were posted on the CDS and SPIRES websites throughout the survey and for two weeks on the website of another major journal, Physical Review D. Information on the survey was also appended for two weeks to the correspondence between the editors and the authors of a third major journal, the European Physical Journal C.

Table 1 presents the distribution of the respondents per country, which confirms the worldwide character of the HEP community and the worldwide spread of the survey. The fraction of answers per country mostly follows the distribution per country of the HEP authorship, as estimated in References 16 and 17, further confirming the absence of systematic biases in the response to the survey. The only appreciable trend is a reduced participation to the survey from Asia. Japan, China, Korea and Taiwan account for about 15\% of HEP authors [17], but they only comprise $5 \%$ of the respondents to the survey.

Table 2 presents the distribution of the respondents per field of activity, with theorists accounting for about $60 \%$ of the total respondents. Table 3 presents the experience of the respondents in the field, $76 \%$ have been active HEP scholars for 6 years or more. These data reflect well the demographics of the HEP community observed in the SPIRES HEPNames database [18]. The respondents to the survey are heavy users of HEP information resources: as presented in Table 4, $82 \%$ use such resources a few times a week or more.

\section{Preferred Systems}

The first question asked in the survey was: Which HEP information system do you use the most? The question, as all other questions discussed in this section, did not allow multiple choices, offering "radio buttons" for arXiv, CDS, Google, Google Scholar and SPIRES, as well as a free-text box for entering the name of another system. $1 \%$ of the respondents made use of this last possibility, mostly to refer to ADS, or to name two systems, typically arXiv and SPIRES, confirming the perception of these two as a single entity.

The results are presented in Table 5 and Figure 1. Community-based systems, comprising ADS, arXiv, CDS, SPIRES and local library services, are the platform of choice for over $91 \%$ of the respondents. The combination of SPIRES and arXiv represents the vast majority of this fraction. $9 \%$ of the respondents use Google or Google Scholar, while commercial systems see a negligible use, around $0.1 \%$.

An interesting correlation with the seniority of HEP scholars is observed, whereby Google is the system of choice for $6 \%$ of scholars active in the field for 10 years or more, but for $22 \%$ of scholars active in the field for 2 years or less. This trend is presented in Figure 2. It should be noted that the use of Google or Google Scholar benefits strongly from the fact that community-based systems have made their content available for harvesting. At the same time, Google and Google Scholar also act, as in many other fields, as a broader alternative to publisher portals, given that indexing of many publisher websites has taken place in recent years. 
Six further questions were asked to assess the use of different resources according to the tasks at hand: Which HEP information system do you use the most to find...

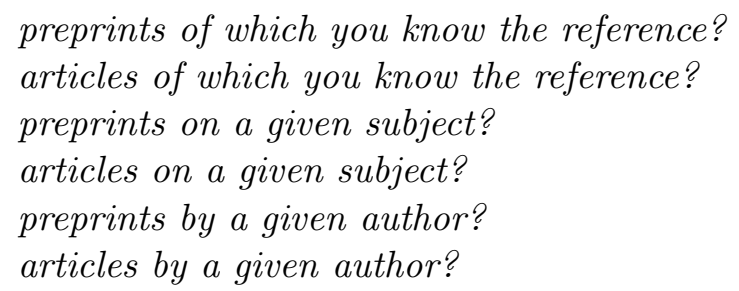

The answers are summarized in Table 5. These more specific questions reveal that the respondents change their behavior based on the task at hand, rather than operating only out of loyalty to a particular system. In particular their changes in usage seem to match some of the notable features of the available systems. As before a trend for a larger usage of Google by younger scholars is detected for these six questions, as summarized in Table 6 .

Figure 3 presents an aggregation of the use of community-based services, Google and commercial systems for the answers concerning searches for preprints and searches for articles. Again, community-based services dominate.

As expected, the maximum usage of commercial services, and in particular publishers' websites, is observed in searches for articles whose reference is known. However, this number stays remarkably low, at $4.5 \%$, confirming that community-based services are the preferred gateway to information, including the published literature, for HEP scholars.

It is also interesting to remark that arXiv figures as the second favorite service in searches for articles. This is somewhat surprising as the site mission is the dissemination of preprints. However, HEP scholars routinely submit to arXiv an updated version of their preprints in an author-formatted post-peer-review version, and therefore make arXiv also a resource for published literature. Moreover, as the HEP content of arXiv is fully indexed by SPIRES, and for many users the distinction between the two is blurred, a user searching for a bibliographic reference in SPIRES, who clicks on the link to the arXiv version rather than the publisher version, would be inspired to answer that arXiv is her system of choice for such a search.

In general, arXiv and SPIRES answer the needs of the vast majority of users, who do recognize the relative strengths and weaknesses of these two services as they move back and forth between them according to the task at hand. SPIRES is favored for journal literature, while arXiv increases its direct usage when preprints are desired. SPIRES is also more heavily favored when searching by author, possibly due to its more advanced author search and better author data. Subject searching, especially for published articles, sees a dramatic rise in the use of Google, possibly because a broader search may be desired, and the community-based systems do not have the breadth of coverage that Google has. There are several possible explanations for the lack of use of commercial systems: few institutions can afford access to them; if such access exists, most HEP scholars are not aware of such a possibility; even if they are, these systems do not provide detailed information specific to HEP users; even if such information is available, it is often lost within the "noise" of literature from many other fields.

A final question on the preferred systems was: Which HEP information system do you use the most to find theses? The corresponding answers are presented in Table 5 and plotted in Figure 4. Unsurprisingly, Google has a larger share than for any other task, at about 1/3. 
However the efforts of community services to track and index theses is still reflected in $2 / 3$ of the users preferring these services for accessing theses. Commercial systems, again, have a negligible share, around $0.1 \%$.

\section{$5 \quad$ Important Features}

In addition to inquiring about the most heavily used systems for different tasks, the survey aimed to assess the importance of various aspects of information resources. Respondents were asked to tag the importance of 12 features of an information system on a five-step scale, ranging from "not important" to "very important", these features are:

Access to full text
Citation analysis
Collaborative tools
Depth of coverage
Keywords and classification
Multimedia content
Personalization
Quality of content
Search accuracy
Speed to find what you want
Submission interface
User friendliness

The results are presented in Table 7 and summarized in Figure 5. Notably, most features are felt to be important. 9 out of the 12 features were found to be important to over half of the respondents. Even multimedia content, the lowest rated feature, was found to be important by $20 \%$ of the users.

Against this background of important features, access to full-text stood out clearly as the most valued feature, with only 5 respondents of the 1700 who answered this question rating it as not important. Following close behind full-text access are depth of coverage, quality of content and search accuracy. Citation analysis, a feature of many of the systems listed, was further down the list for most users. It was still considered important by most users, but it was clearly a secondary feature, along with user friendliness.

Even if not all systems offer all these features, the perceived importance of each feature was found to be mostly independent from the system most used by the respondents.

The survey included another set of questions, which were clearly labeled as optional, to further understand which additional features are considered important. Out of these 21 additional features, 12 are particularly relevant and are discussed in the following:

\footnotetext{
Access

Finding theses

Finding conference proceedings

Finding articles cited with a given article

Finding articles citing a given articles

Finding top-cited articles by subject
} 


\section{Community}

Finding conference announcements

Annotating and commenting on documents

Directory of authors and affiliations

Retrieving list of publications

\section{Authorship}

Retrieving and exporting article references

Possibility of submitting article revisions

Knowing how often your articles are read

The first five features concentrate on the access to information, the second four are part of a wider service to the community, while the last three are services tailored to authors. Respondents were asked to tag the importance of these features on a five-step scale, ranging from "not important" to "very important". The results are presented in Table 8 and Figure 6. Some of the services which are felt as moderately or very important by most respondents, such as the possibility of finding all articles citing a given article and the possibility of submitting a revised version of an article, are currently offered by SPIRES and arXiv, respectively. It is interesting to note that two of the other features which are perceived as moderately or very important by most respondents, the possibility of finding all articles cited with a given article and the possibility of knowing how often an article or preprint is downloaded, are not currently offered by the most widespread services.

\section{Winds of Change}

The survey explicitly inquired about the level of change that HEP scholars would expect and require from their information resources: $75 \%$ expected "some" to "a lot of" change in the next five years, while only $12 \%$ expected no change ${ }^{4}$.' To structure this perception of change, respondents were asked to imagine their ideal information system in five years and tag the importance of 11 possible features on a five-step scale from "not important" to "very important". These features are:

Access from your PDA

Access to data in figures and tables

Authoring tools

Centralization ${ }^{5}$

Collaborative tools

Connections to fields outside HEP

Inclusion of multiple types of documents

Linked presentation of all instances of a result ${ }^{6}$

Multimedia content

Personalization

Recommendation of documents of potential interest

\footnotetext{
${ }^{4} 90 \%$ of respondents answered this question.

${ }^{5}$ The full-text of the "Centralization" feature was "Centralization: one single portal to all the information".

${ }^{6}$ The full-text of the "Linked presentation" feature was "Linked presentation of all instances of a result, from notes to theses, from conference slides to articles".
} 
The results are presented in Table 9 and Figure 7. While "modern" features such as multimedia content or access from a PDA were not considered overwhelmingly important, about $90 \%$ of the users tagged three features as important: the linked presentation of all instances of a result, the centralization and the access to data in figures and tables. Immediately following these three is the extension of the level of service of HEP information systems to other, related, disciplines. The last is hardly surprising: SPIRES has since long bridged the divide towards astrophysics, cosmology and nuclear physics, following an increased interdisciplinary activity of HEP scholars.

A final question tried to assess the potential for the implementation of Web2.0 features to capture user-tagged content. Respondents were asked: If a simple web interface would show you an article and offer a set of categories to which it could belong, how much time would you spend in this tagging system to give a service to the community? Of the $90 \%$ of respondents who answered this question, $19 \%$ would not spend any time on this system, while $63 \%$ would spend between five minutes a day and an hour a week. The breakdown of these answers by the seniority of the respondents is presented in Figure 8. There is an immense potential for user-generated, or rather user-tagged and user-curated, content in the field of information provision in HEP, as in many other fields of web-based communication.

\section{Conclusions}

The response to the survey was overwhelming, with over 2,000 HEP scholars, representing about $10 \%$ of the community, answering basic and long questions, sharing their appreciation and vision for information management in the field. The large participation is per se a result, signifying the engagement of the community with its information resources.

The main finding of the survey is that community-based services are overwhelmingly dominant in the research workflow of HEP scholars. Although the popularity of Google increases with younger researchers, the field-specific utility provided by these highly-tailored services is perceived as more relevant. Commercial systems are virtually unused in the field.

While the various community-based systems have stronger and weaker features, users attach paramount importance to three axes of excellence: access to full-text, depth of coverage and quality of content.

Future evolution of these systems should be charted by the clear desire of users for a centralized and coherent presentation of all instances of a scientific result, with access to data in figures and tables and a connection to fields outside of HEP. The survey shows that there exists a remarkable potential for capturing user-tagged content, with a large fraction of users willing to invest time in such a community service.

The survey collected thousands of free-text answers about the most and least liked features of current systems and the user requirements for future evolution of information provision in the field. While a detailed study of these additional data is underway, and outside the scope of this Article, some inspiring answers are distilled in the Appendix.

The results discussed in this Article confirm the exceptional situation of the HEP community in the field of scholarly communication: decades of efforts in developing, maintaining, populating and curating community-based services enable an efficient research workflow for HEP scientists and are met by overwhelming user loyalty. Scholarly communication is at the dawn of a new era, with the onset of institutional repositories and author self-archiving of 
research results. In this evolving landscape, could the decades-old success story of communitybased HEP information systems, and their discipline-based content aggregation, provide inspiration for scholarly communication in other fields?

\section{Acknowledgments}

First and foremost we wish to thank all HEP scholars who answered this survey, sharing their opinions, suggestions, wishes and constructive criticism on HEP information systems. We are grateful to our colleagues who shared their insight in the field of information management, which were crucial in the preparation of the survey: Catherine Cart, Jocelyne Jerdelet, JeanYves Le Meur, Tibor Simko, Tim Smith, and Jens Vigen at CERN; Zaven Akopov and Kirsten Sachs at DESY; and Pat Kreitz and Ann Redfield at SLAC. This study would not have reached such a large audience without the collaboration of Paul Ginsparg and Simeon Warner at arXiv, Enrico Balli at SISSA/Medialab, Bob Kelly and Erick Weinberg at APS and Christian Caron at Springer, who kindly disseminated information about the survey, and to whom we are indebted. 


\section{Appendix: Inspiring Free-Text Answers}

In addition to the results presented above, the survey collected thousands of free-text answers, inquiring about features of current systems and their most-desired evolution. A detailed study of these comments is underway and outside the scope of this Article. However, it is particularly interesting to distill some of these answers here, in order to complete the assessment of the engagement of the HEP community with the systems which serve its information needs and its expectations for future developments. Some of the most inspiring free-text answers were along the following lines:

- Desire for seamless open access to older articles, prior to the onset of arXiv in the '90s.

- Improved full-text search and access to research notes of large experimental collaborations. These are a crucial gray-literature channel where large amounts of information and details about the results of large experiments transit.

- Indexing of conference talks and long-term archiving of the corresponding slides, beyond the lifetime of conference websites. Interlinking of these slides with the corresponding conference proceedings, in preprint form with reference to published volumes, and possibly other instances describing the results.

- Use of the HEP information resources as fora for the publication of ancillary material, crucial in the research workflow, and in particular:

- numerical data corresponding to tables;

- numerical data corresponding to figures;

- correlation matrices and additional information beyond these presented in tables, to allow an effective re-use of scientific results;

- fragments of computer code accompanying complex equations in articles, to improve the research workflow and reduce the possibility of errors;

- primary research data in the form of higher-level objects.

- "Smarter" search tools, giving access to articles related to articles of interest.

- Establishment of some new sort of open peer-review, overlaid on arXiv. 


\section{References}

[1] L. Goldschmidt-Clermont, Communication Patterns in High-Energy Physics, 1965; published in High Energy Physics Libraries Webzine, issue 6, March 2002. http://library.cern.ch/HEPLW/6/papers/1/.

[2] L. Addis, http://www.slac.stanford.edu/spires/papers/history.html [Last visited March 4, 2008].

[3] T. Berners-Lee, Weaving the Web, HarperCollins, San Francisco (1999).

[4] P. Ginsparg, Computers in Physics 8 (1994) 390.

[5] http://www.slac.stanford.edu/history/earlyweb/history.shtml [Last visited March 4, 2008].

[6] http://cdsware.cern.ch.

[7] http://arxiv.org.

[8] http://cds.cern.ch.

[9] C. O'Dell et al., CERN-OPEN-2003-053 http://doc.cern.ch/archive/electronic/cern/preprints/open/open-2003-053.pdf.

[10] http://www.slac.stanford.edu/spires.

[11] P. A. Kreitz and T. C. Brooks, Sci. Tech. Libraries 24 (2003) 153 [arXiv:physics/0309027].

[12] http://adswww.harvard.edu.

[13] http://google.com.

[14] http://scholar.google.com.

[15] J. Krause and S. Mele, in preparation.

[16] S. Mele et al., Journal of High Energy Physics 12 (2006) S01 [arXiv:cs.DL/0611130].

[17] J. Krause, C. Lindqvist and S. Mele, CERN-OPEN-2007-014 http://doc.cern.ch/archive/electronic/cern/preprints/open/open-2007-014.pdf.

[18] http://www.slac.stanford.edu/spires/hepnames. 


\begin{tabular}{|l|r|l|r|}
\hline Country & Fraction & Country & Fraction \\
\hline United States & $27.4 \%$ & Iran & $0.9 \%$ \\
Germany & $9.5 \%$ & Mexico & $0.9 \%$ \\
Italy & $7.7 \%$ & Australia & $0.8 \%$ \\
United Kingdom & $6.5 \%$ & Denmark & $0.8 \%$ \\
CERN & $4.9 \%$ & Sweden & $0.8 \%$ \\
France & $4.1 \%$ & Greece & $0.8 \%$ \\
India & $3.4 \%$ & Portugal & $0.8 \%$ \\
Spain & $3.0 \%$ & Argentina & $0.7 \%$ \\
Canada & $2.6 \%$ & Korea & $0.7 \%$ \\
Brazil & $2.4 \%$ & Austria & $0.6 \%$ \\
Russia & $2.4 \%$ & Poland & $0.6 \%$ \\
Switzerland & $2.2 \%$ & Chile & $0.5 \%$ \\
China & $2.1 \%$ & Finland & $0.5 \%$ \\
Japan & $1.7 \%$ & Taiwan & $0.5 \%$ \\
Israel & $1.5 \%$ & Czech Republic & $0.3 \%$ \\
Netherlands & $1.2 \%$ & Norway & $0.2 \%$ \\
Belgium & $1.1 \%$ & Hungary & $0.2 \%$ \\
Turkey & $0.9 \%$ & Others & $4.8 \%$ \\
\hline
\end{tabular}

Table 1: Distribution of answers per country. Users based at CERN were asked to indicate "CERN" and not "Switzerland". 97\% of respondents answered this question.

\begin{tabular}{|l|r|}
\hline Field of activity & Fraction \\
\hline Theory & $61.3 \%$ \\
Experiment & $22.2 \%$ \\
Software & $5.5 \%$ \\
Instrumentation & $3.5 \%$ \\
Accelerators & $2.7 \%$ \\
Engineering & $1.3 \%$ \\
Others & $3.5 \%$ \\
\hline
\end{tabular}

Table 2: Field of activity of respondents to the survey. $96 \%$ of respondents answered this question. 


\begin{tabular}{|l|r|}
\hline How long have you & \\
used HEP search engines? & Fraction \\
\hline >10 years & $45.9 \%$ \\
6-10 years & $30.0 \%$ \\
3-5 years & $18.7 \%$ \\
0-2 years & $5.4 \%$ \\
\hline
\end{tabular}

Table 3: Experience of respondents. 95\% of respondents answered this question.

\begin{tabular}{|l|r|}
\hline How frequently do you & \\
use HEP search engines? & Fraction \\
\hline Every day & $57.0 \%$ \\
A few times per week & $25.6 \%$ \\
Once a week & $5.5 \%$ \\
A few times per month & $7.4 \%$ \\
Once a month & $2.0 \%$ \\
A few times a year & $2.5 \%$ \\
\hline
\end{tabular}

Table 4: Frequency of use of HEP search engines. 95\% of respondents answered this question. 


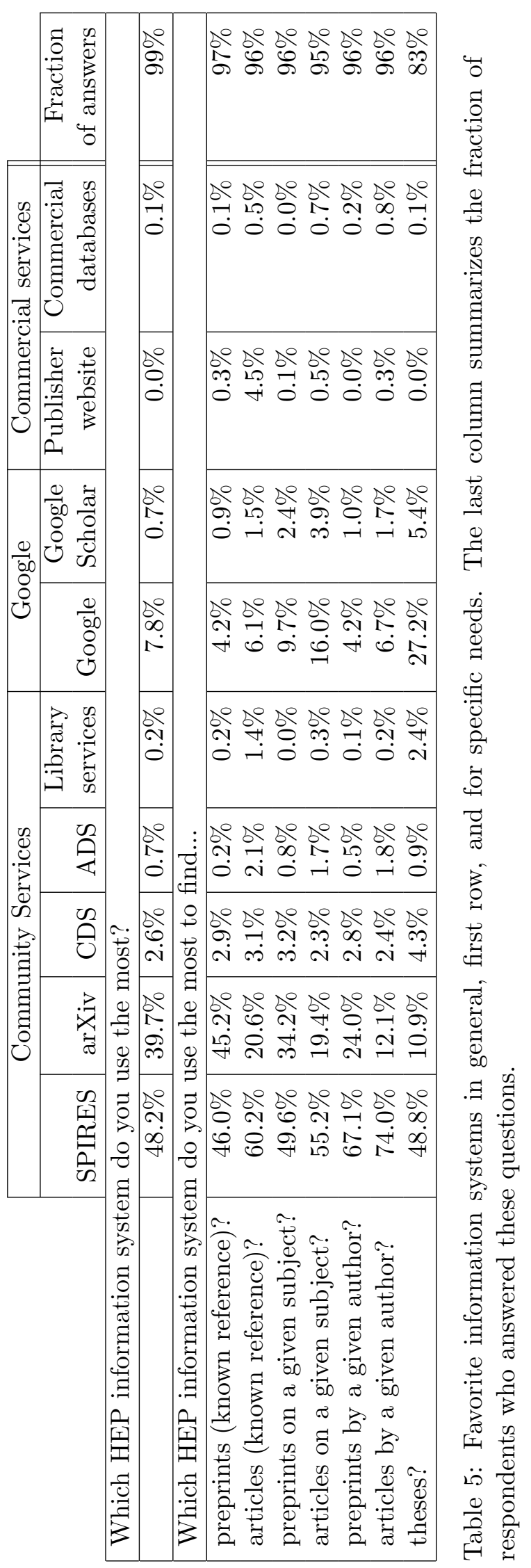




\begin{tabular}{|l|r|r|}
\cline { 2 - 3 } \multicolumn{1}{c|}{} & \multicolumn{2}{c|}{$\begin{array}{c}\text { Fraction of users of } \\
\text { Which system do you use }\end{array}$} \\
\cline { 2 - 3 } the most... & $>10$ years & $<2$ years \\
\hline in absolute & $6.0 \%$ & $22.1 \%$ \\
for preprints (known reference)? & $3.2 \%$ & $15.4 \%$ \\
for articles (known reference)? & $5.4 \%$ & $16.2 \%$ \\
for preprints on a given subject? & $9.3 \%$ & $29.5 \%$ \\
for articles on a given subject? & $17.5 \%$ & $34.4 \%$ \\
for preprints by a given author? & $3.1 \%$ & $17.0 \%$ \\
for articles by a given author? & $5.9 \%$ & $20.1 \%$ \\
for theses? & $30.0 \%$ & $41.0 \%$ \\
\hline
\end{tabular}

Table 6: Penetration of Google and Google Scholar as information resources in HEP as a function of the seniority of the scholars. 


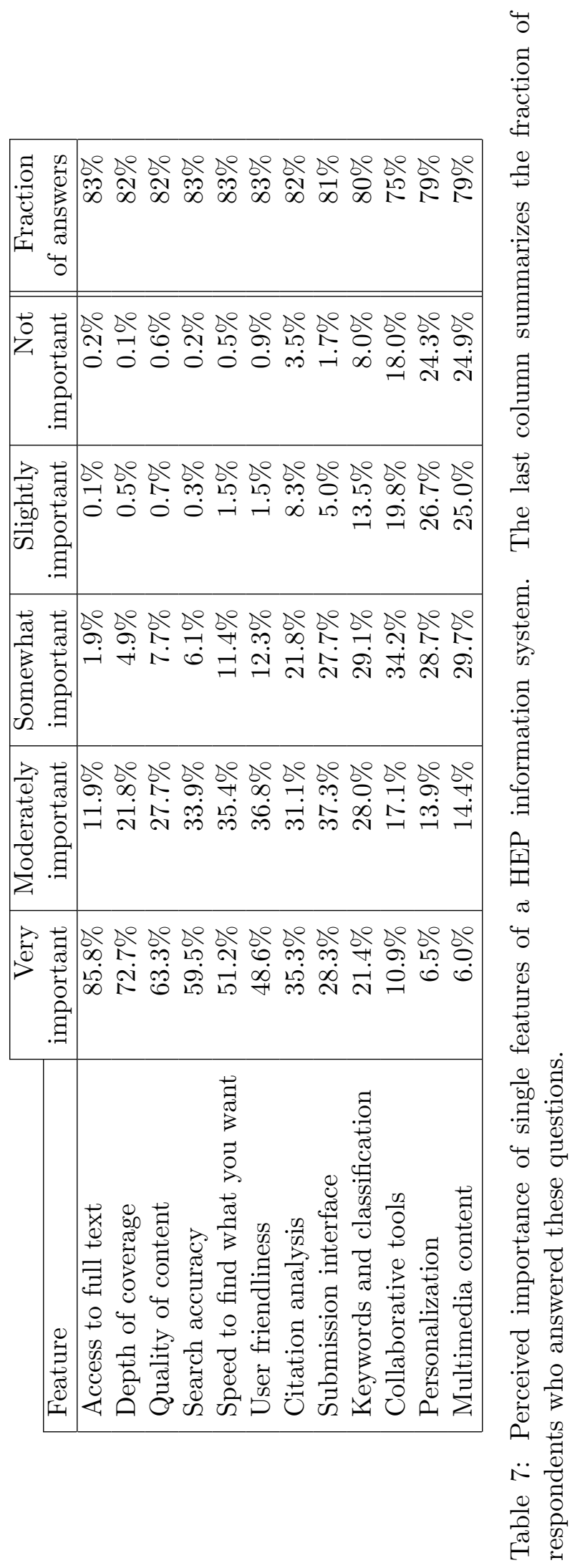




\begin{tabular}{|c|c|c|c|}
\hline 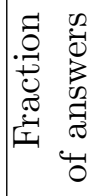 & 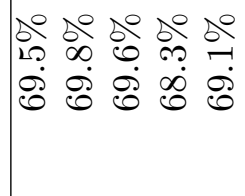 & 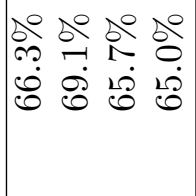 & 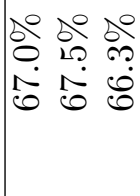 \\
\hline 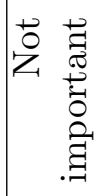 & 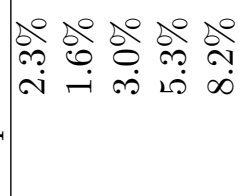 & 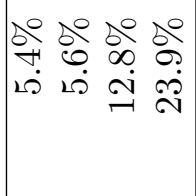 & 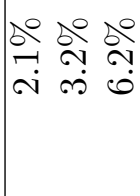 \\
\hline 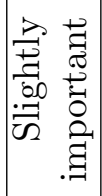 & 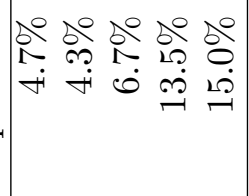 & 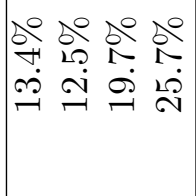 & 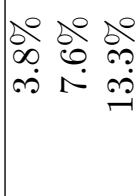 \\
\hline 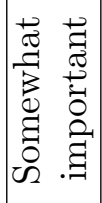 & 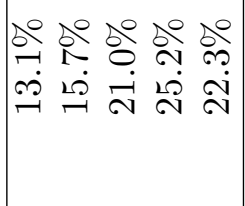 & 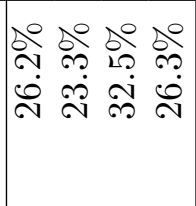 & 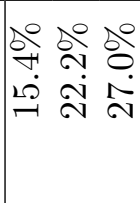 \\
\hline 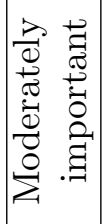 & 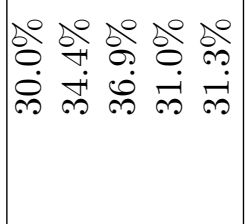 & 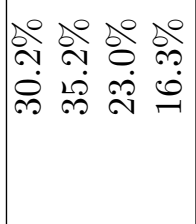 & 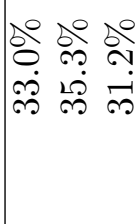 \\
\hline 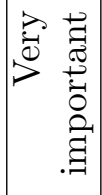 & 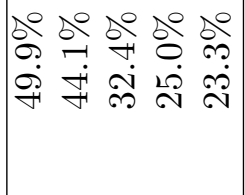 & 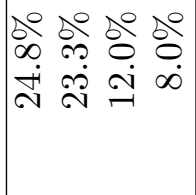 & 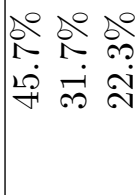 \\
\hline 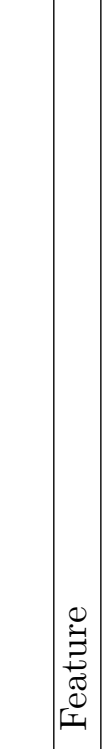 & 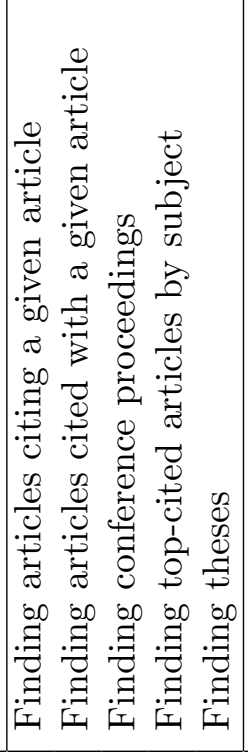 & 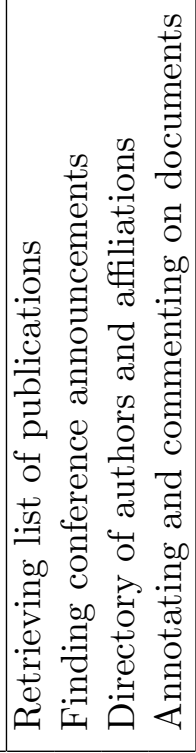 & 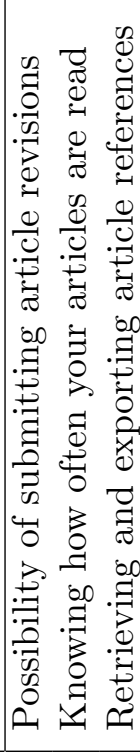 \\
\hline
\end{tabular}

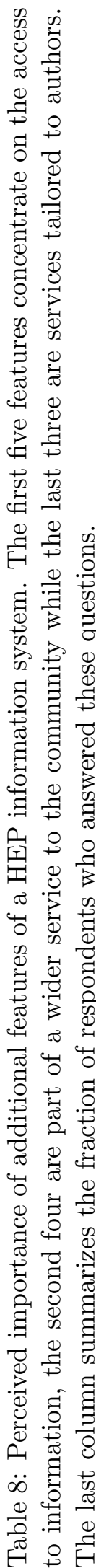




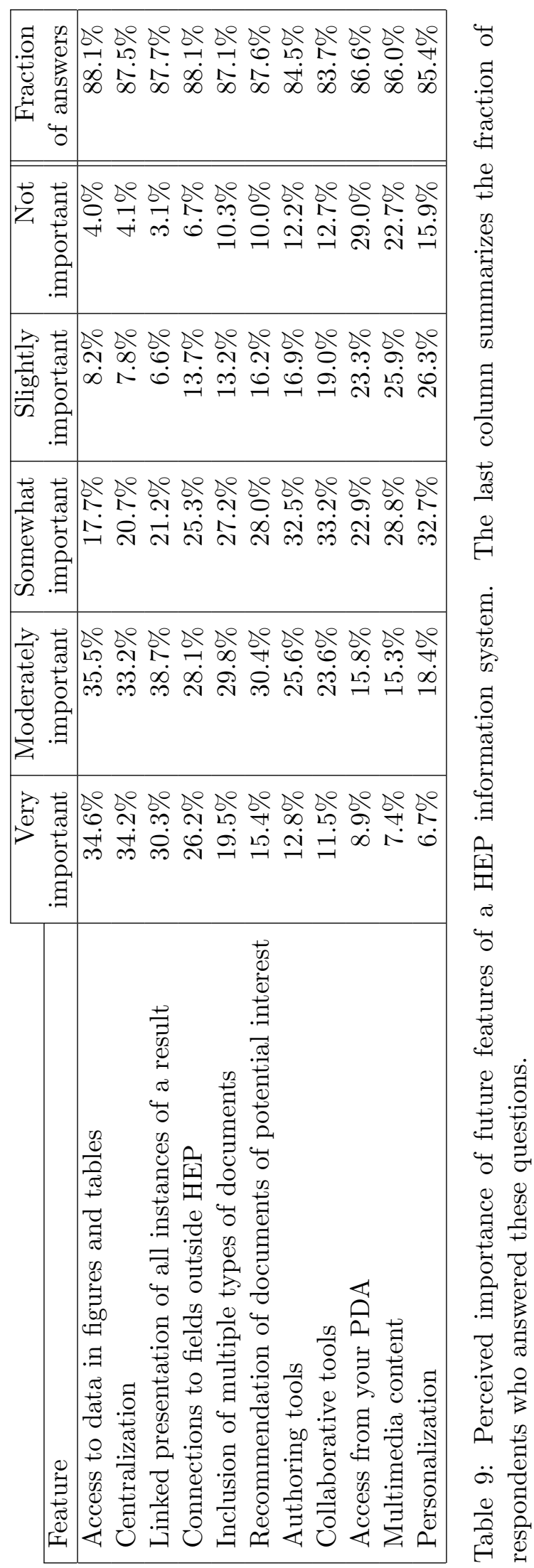


Which HEP information system do you use the most?

\section{Community-based systems (91.4\%)}

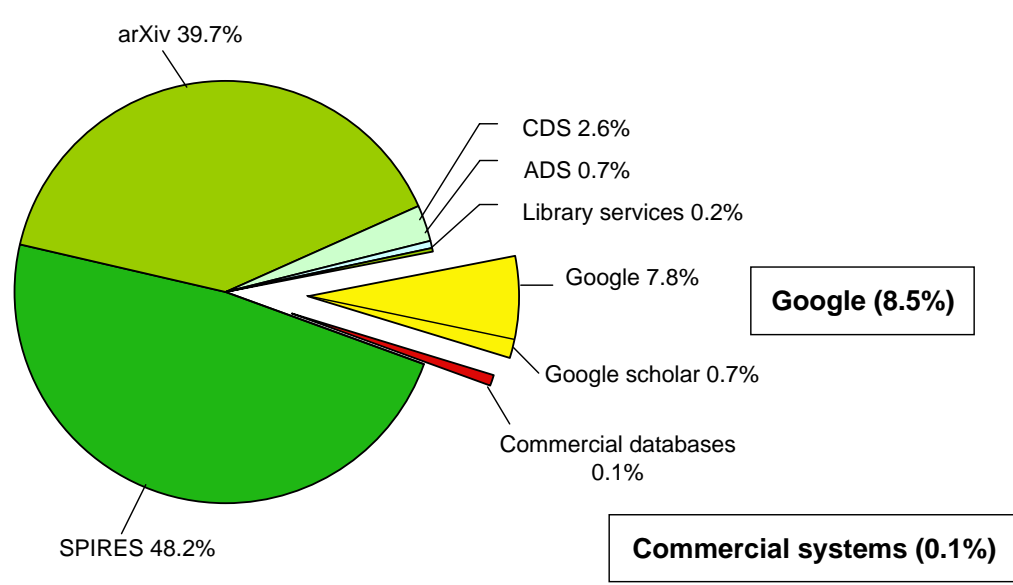

Figure 1: Favorite information resources for HEP scholars. The slice corresponding to commercial systems is enlarged for increased visibility. 
Which HEP information system do you use the most?

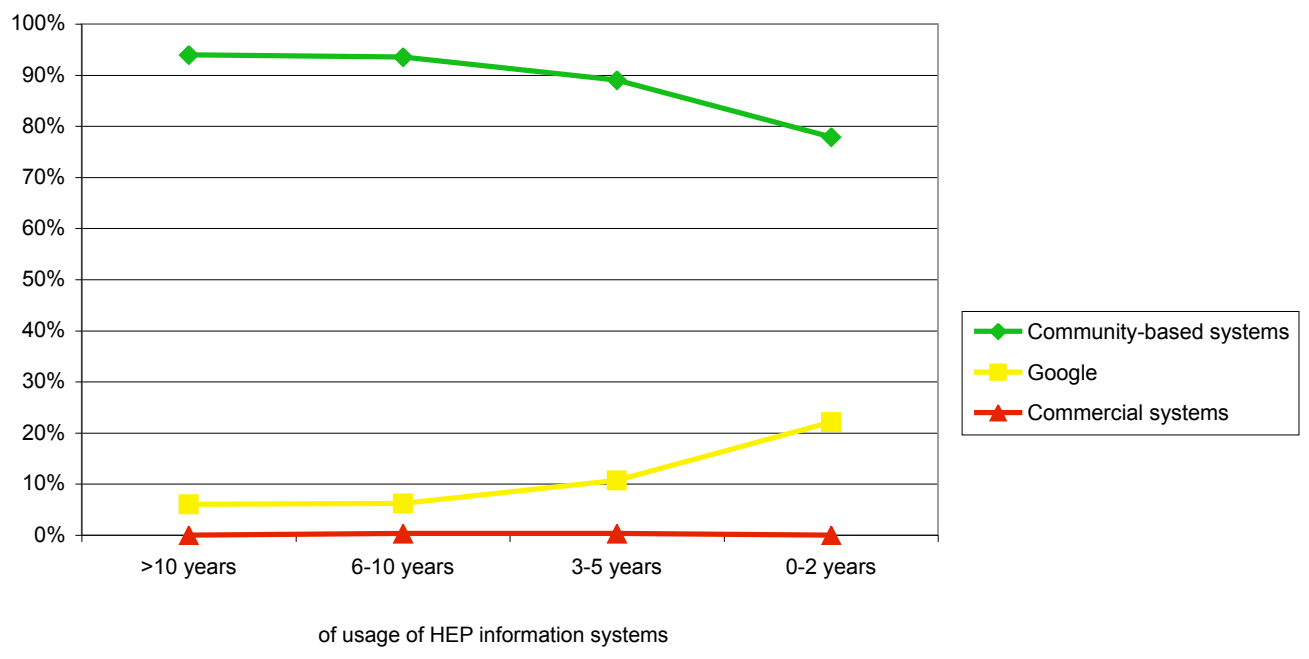

Figure 2: Categories of information resources used by HEP scholars as a function of their seniority in the field. 

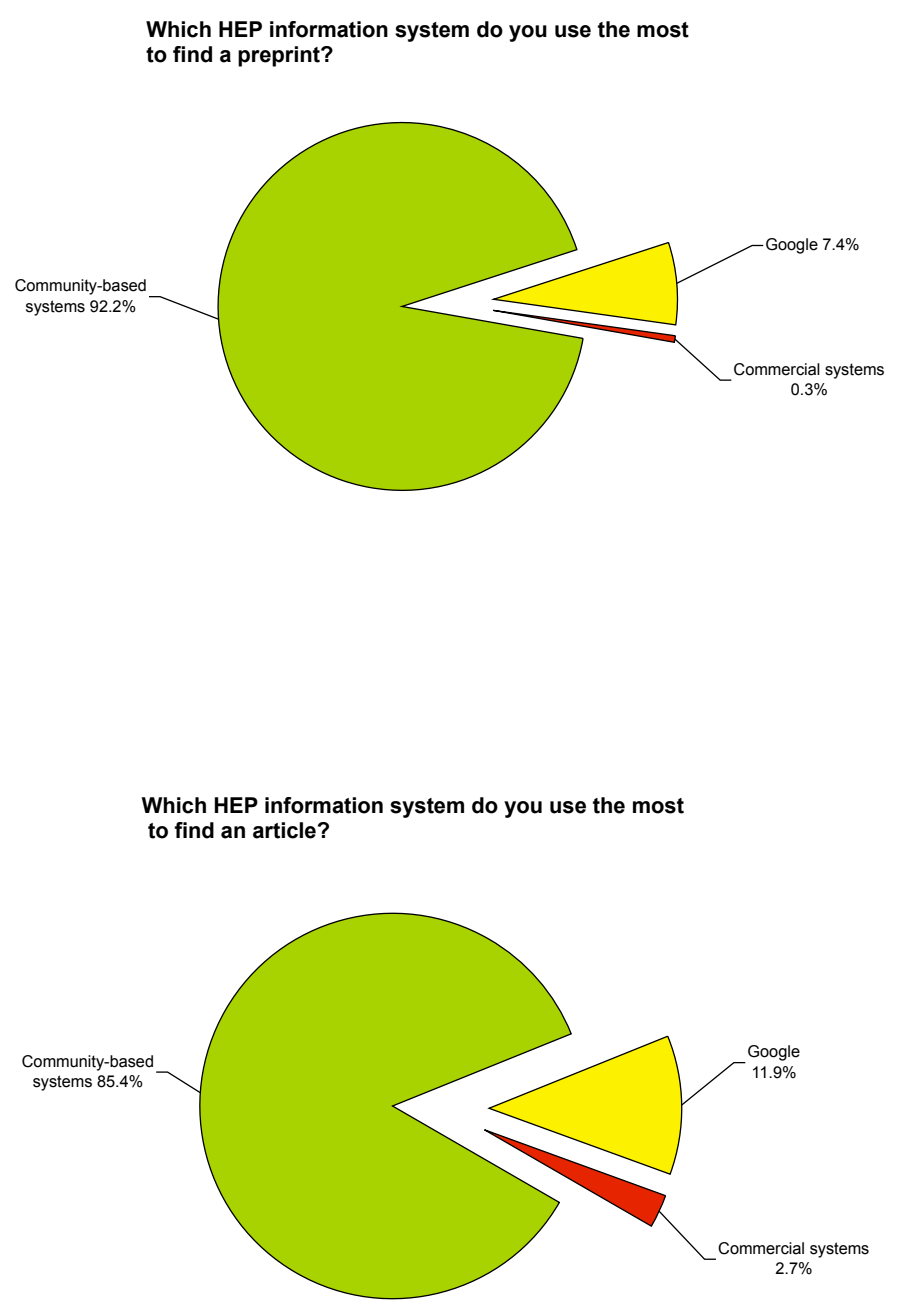

Figure 3: Type of information resource most used by HEP scholars to access information in the form of preprints or published articles. 


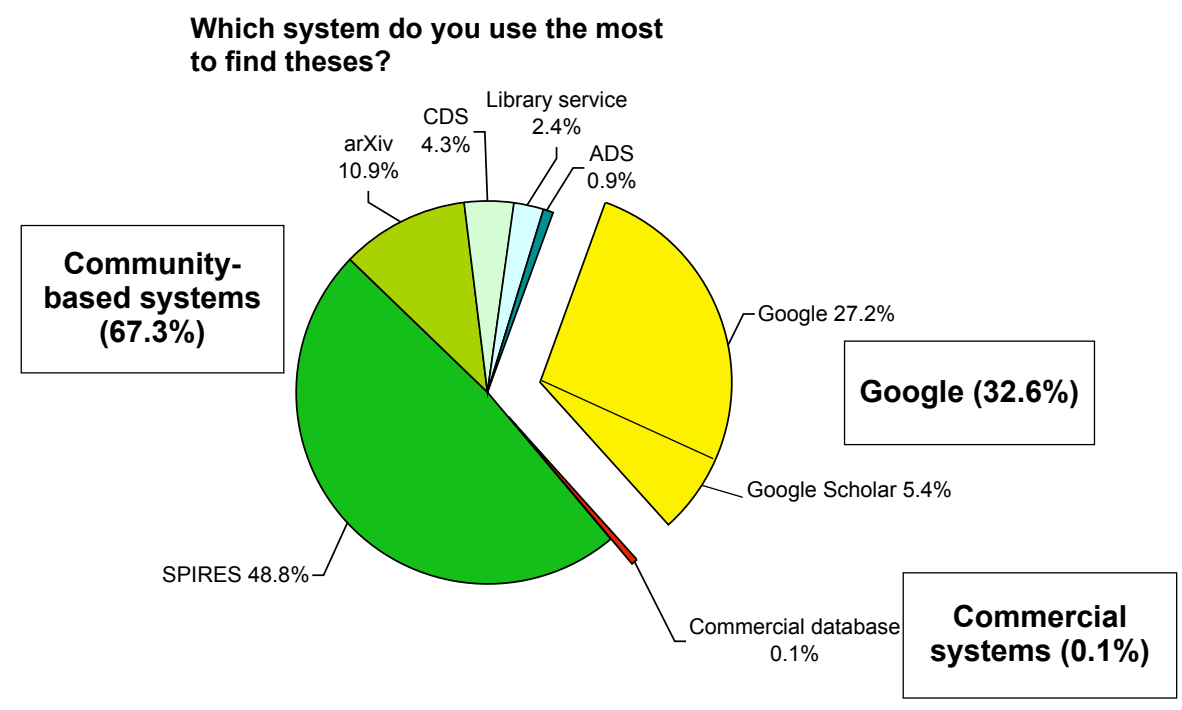

Figure 4: Information resources most used by HEP scholars to search for theses. The slice corresponding to commercial systems is enlarged for increased visibility. 


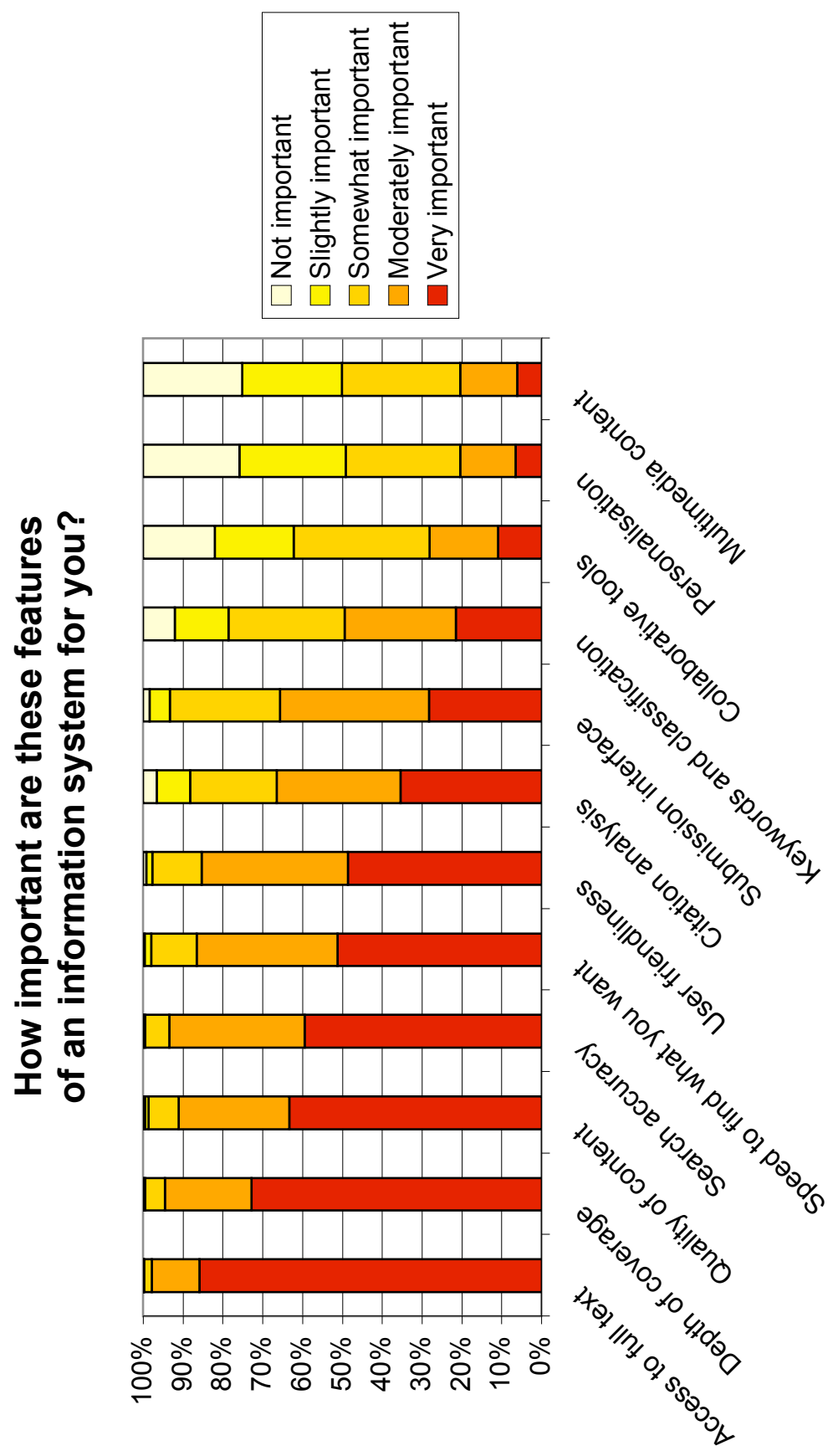

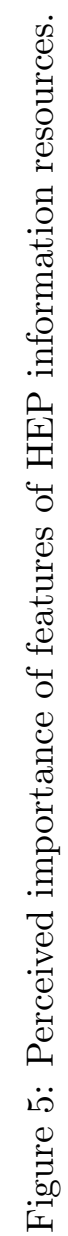




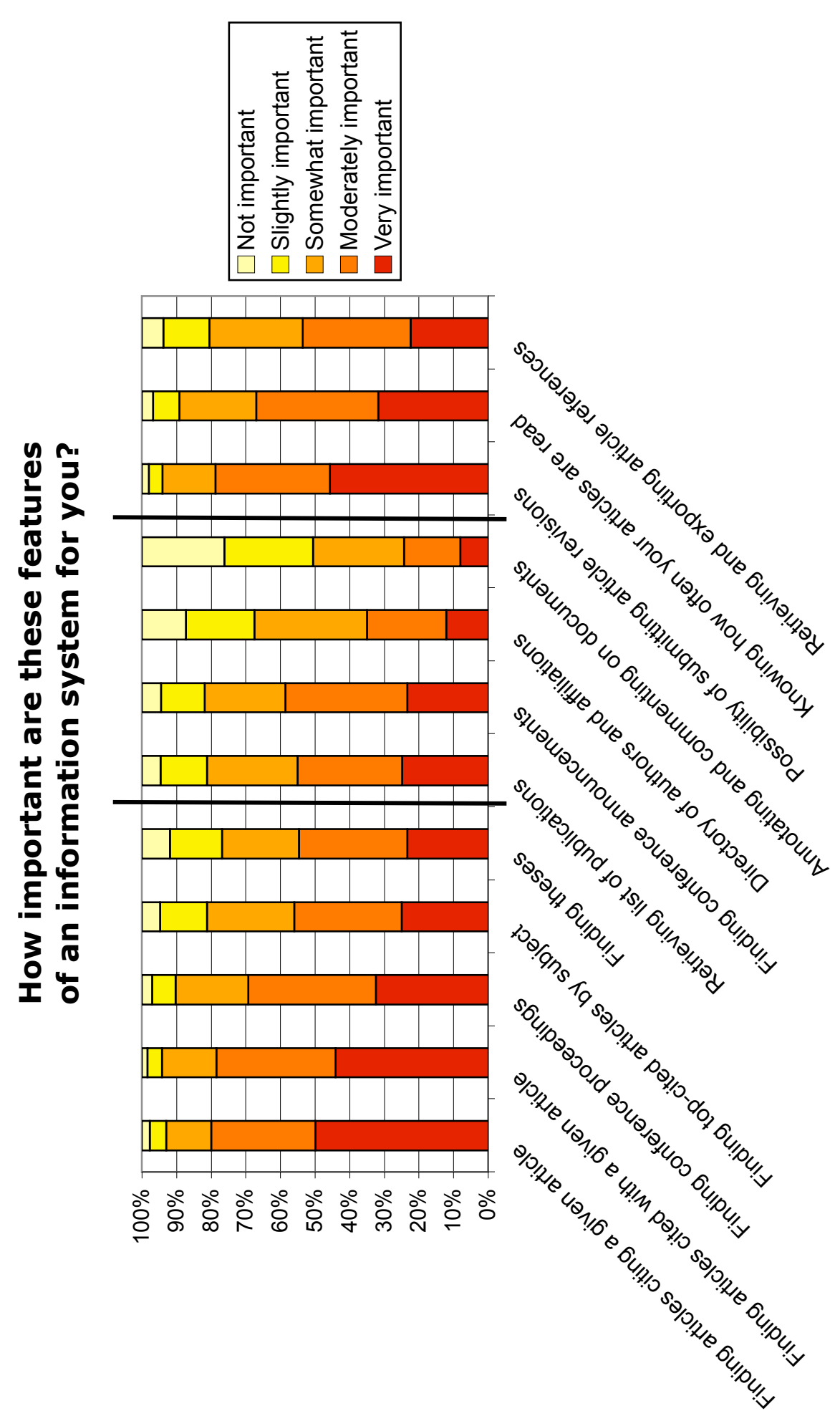

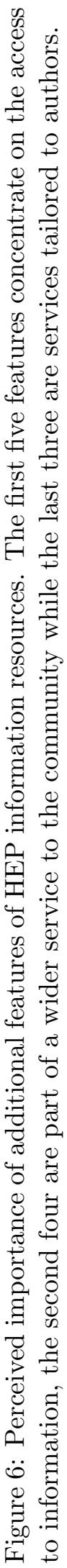



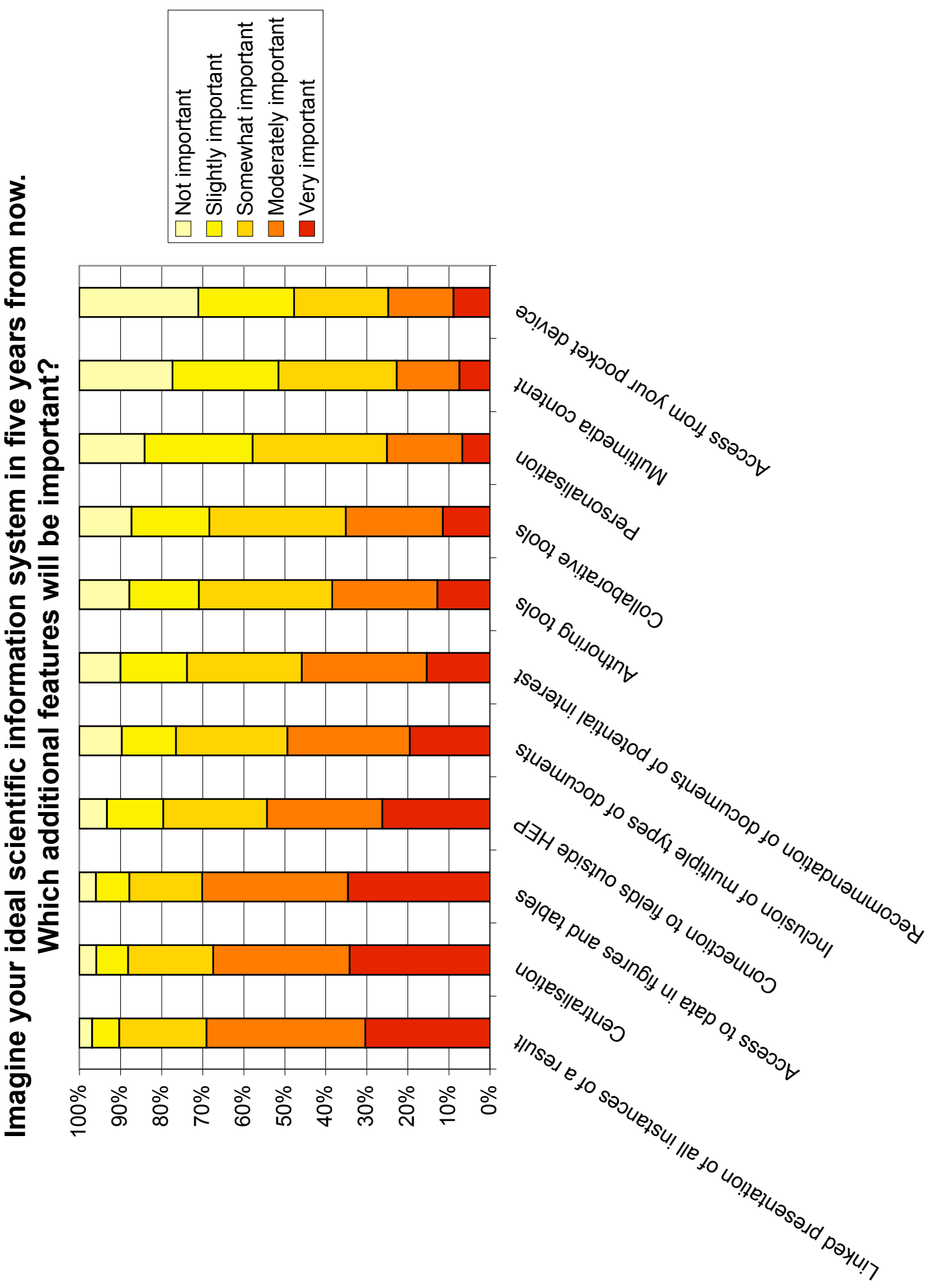

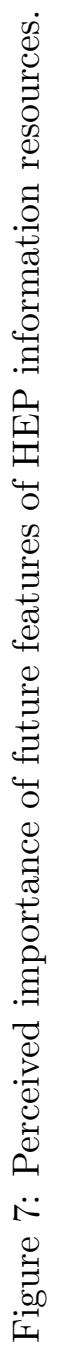




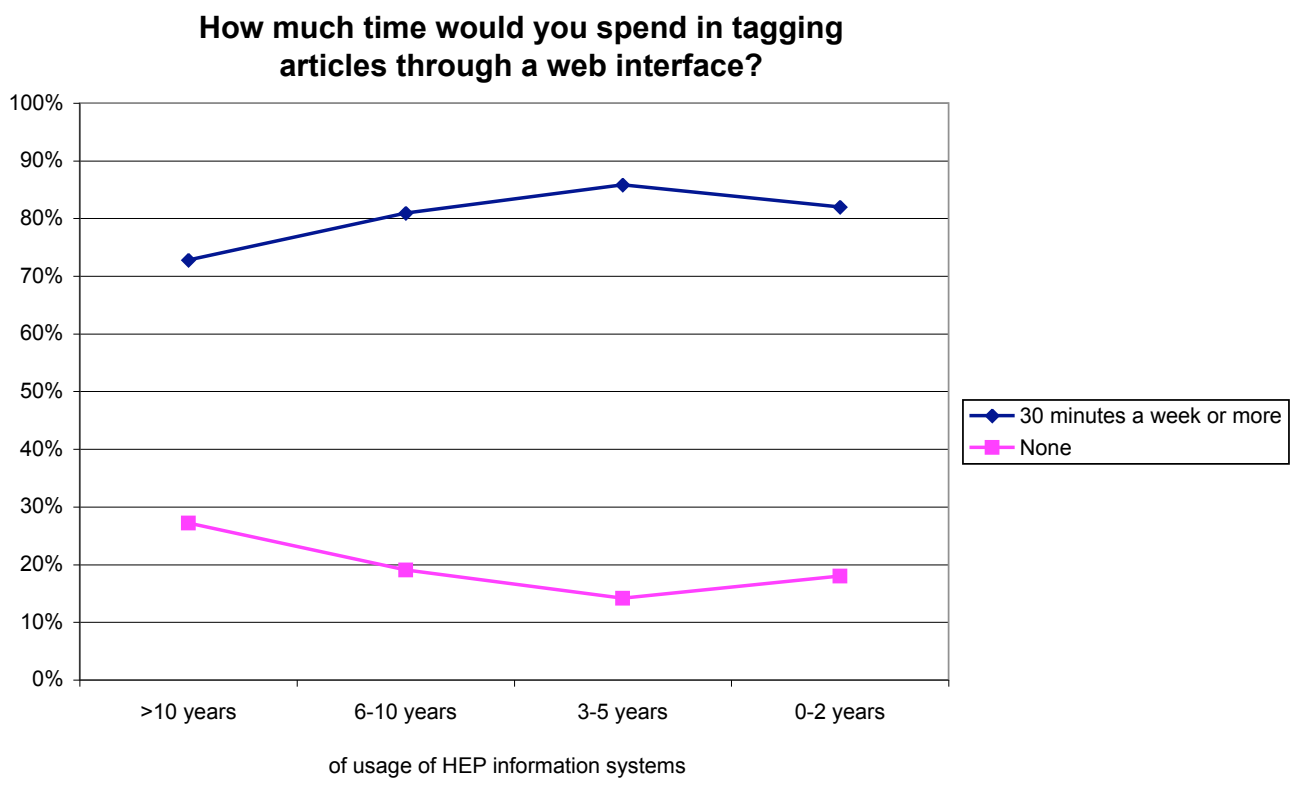

Figure 8: Interest in participating in user-tagging of content as a function of the seniority of respondents. 